\title{
From Single Grains to Texture
}

\section{By Kun Yan, Klaus-Dieter Liss*, Ulf Garbe, John Daniels, Oliver Kirstein, Huijun Li and Rian Dippenaar}

Structural materials, such as metals, ceramics, and their composites are most often polycrystalline. The nature, morphology, and composition of their microstructure determine in large measure the mechanical properties of the final product, and the art to design novel materials is to find particular arrangements which make them harder, more shock absorbing, heat resistant, or self-recovering upon damage and aging. The understanding of the basic processes and their interplay in a polycrystalline structure are most important for improved simulation of plastic deformation and to predict their thermo-mechanical behavior.

Here we present an in situ study of cold plastic deformation of coarse grained copper, which provides new insights into the real-time evolution of the microstructure. Conventional metallographic design is based on thermo-mechanic simulation and retrospective investigations, usually employing optical and electron microscopy as well as conventional $X$-ray and electron diffraction. These (near-) surface sensitive investigational tools require accurate cutting and preparation of specimens and information of bulk behavior is usually obtained by laborious serial layer by layer sectioning. High-energy $\mathrm{X}$-rays and neutron radiation, however, have the advantage of deep bulk penetration into the material. While the brilliance of synchrotron light allows narrow, well-collimated beams to distinguish between individual crystallites, overall information, and good statistical averages are better obtained with neutrons.

A schematic illustration of the diffraction set-up used in this study is presented in Figure 1. High-energy X-rays ${ }^{[1]}$ with $87.8 \mathrm{keV}$ photons available at Beamline ID15B of the European Synchrotron Radiation Facility were used to probe the bulk of the material. Copper specimens of $\varnothing 3 \mathrm{~mm}$ and $4.5 \mathrm{~mm}$ height with a grain size of $\sim 300 \mu \mathrm{m}$ were positioned in a load frame

[*] Dr. K.-D. Liss, K. Yan,

Dr. U. Garbe, Dr. O. Kirstein

Australian Nuclear Science and Technology Organisation, PMB 1

Menai NSW-2234, Australia

E-mail: kdl@ansto.gov.au; liss@kdliss.de

K. Yan, Prof. H. Li, Prof. R. Dippenaar

Faculty of Engineering, University of Wollongong

Wollongong NSW-2522, Australia

Dr. J. Daniels

European Synchrotron Radiation Facility, BP 220

F-38043 Grenoble, France and diffraction patterns taken every $0.5 \mathrm{~s}$, probed by a $0.1 \times 0.1 \mathrm{~mm}^{2}$ beam while undergoing continuous compression from 4.5 to $2.4 \mathrm{~mm}$. The morphology of the Debye-Scherrer rings shown in Figure 2 was then analyzed to obtain information about the grain statistics, crystallite perfection, and grain orientation. For each time step, the Debye-Scherrer ring of a selected reflection (Fig. 2) was cut at the $9 \mathrm{o}^{\prime}$ clock position (Fig. 1) and straightened into one line in azimuthal angle $\gamma$ as shown in Figure 3, the vertical axis being time.

While only a few spots from individual crystallites occur initially (Fig. 2(a)), continuous but textured rings evolve as deformation proceeds (Fig. 2(b)). Initially, it appears that the single-crystallite reflections broaden azimuthally, due to an increase of mosaic spread, presumably by the creation of subgrains through the development of dislocation

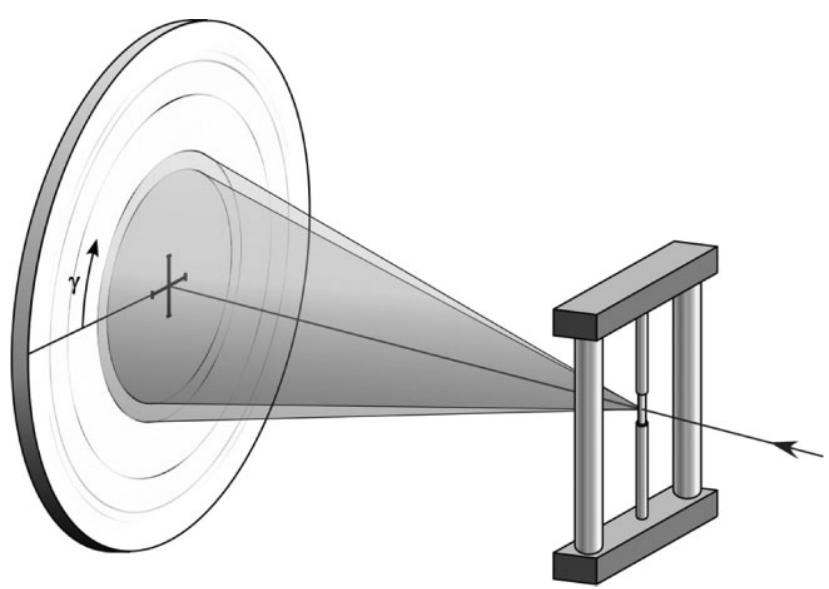

Fig. 1. Schematic illustration of the in situ diffraction set-up. High energy X-rays impinging from the right scatter at the sample while Debye-Scherrer rings with azimuthal angle $\gamma$ are recorded by an online 2D detector in transmission geometry. The cylindrical sample is subjected to external stress by the load frame. 
a)

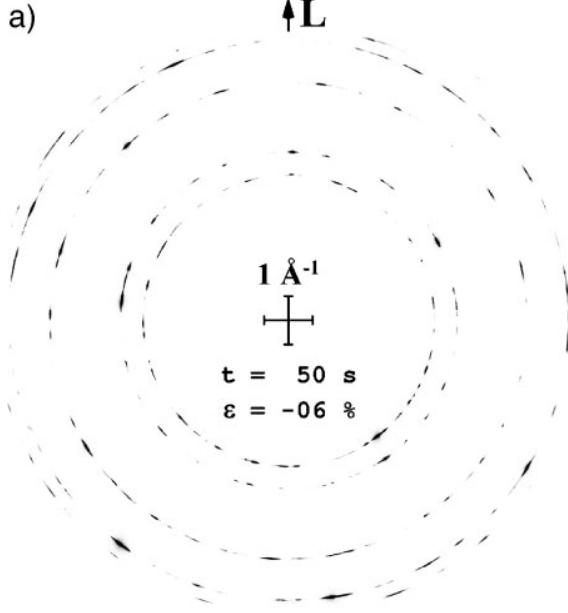

b)

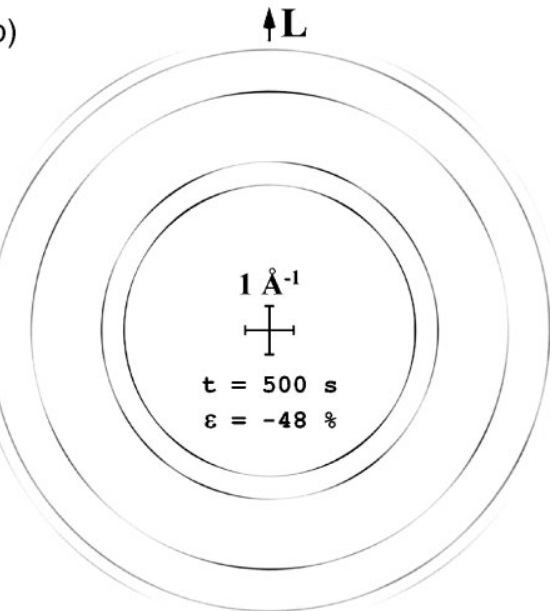

Fig. 2. Two-dimensional X-ray diffraction patterns (dark is high intensity) (a) Following compression to $\varepsilon=-0.06$ spotty Debye-Scherrer rings appear from a small number of crystallites. (b) Upon further plastic deformation $(\varepsilon=-0.48$ in this instance) the spotty rings evolve into continuous, but textured rings.

structures. ${ }^{[2]}$ In a simple model, the mosaic of a single grain broadens conically in both dimensions of orientation space. Grains which originally had reciprocal lattice vectors lying slightly off the Ewald sphere, move into a Laue diffraction condition and reflect. Such a process is represented by the appearance of many reflections after $\sim 40 \mathrm{~s}(\varepsilon \approx-0.04)$ of deformation. Simultaneously, some of the reflections move sideways in azimuthal angle. This behavior is interpreted as a direct observation of grain rotation ${ }^{[3]}$ into the preferred orientation inherent to plastic deformation. At some stage of deformation (after about $100-250 \mathrm{~s} ; \varepsilon \approx-0.06$ to -0.15 ), intensities from neighboring crystallites merge into a continuous Debye-Scherrer ring and single grains cannot be separated any longer. The rings now become continuous, but modulated and subgrains form. Following the compression test, the final texture was measured using neutrons on the Kowari instrument at the OPAL reactor of the Australian Nuclear Science and Technology Organisation. ${ }^{[4,5]}$ The 111 pole figure recalculated from the obtained orientation distribution function is shown in Figure $4 .{ }^{[6]}$ Schematically, the intensity distribution of the 111 Debye-Scherrer ring in

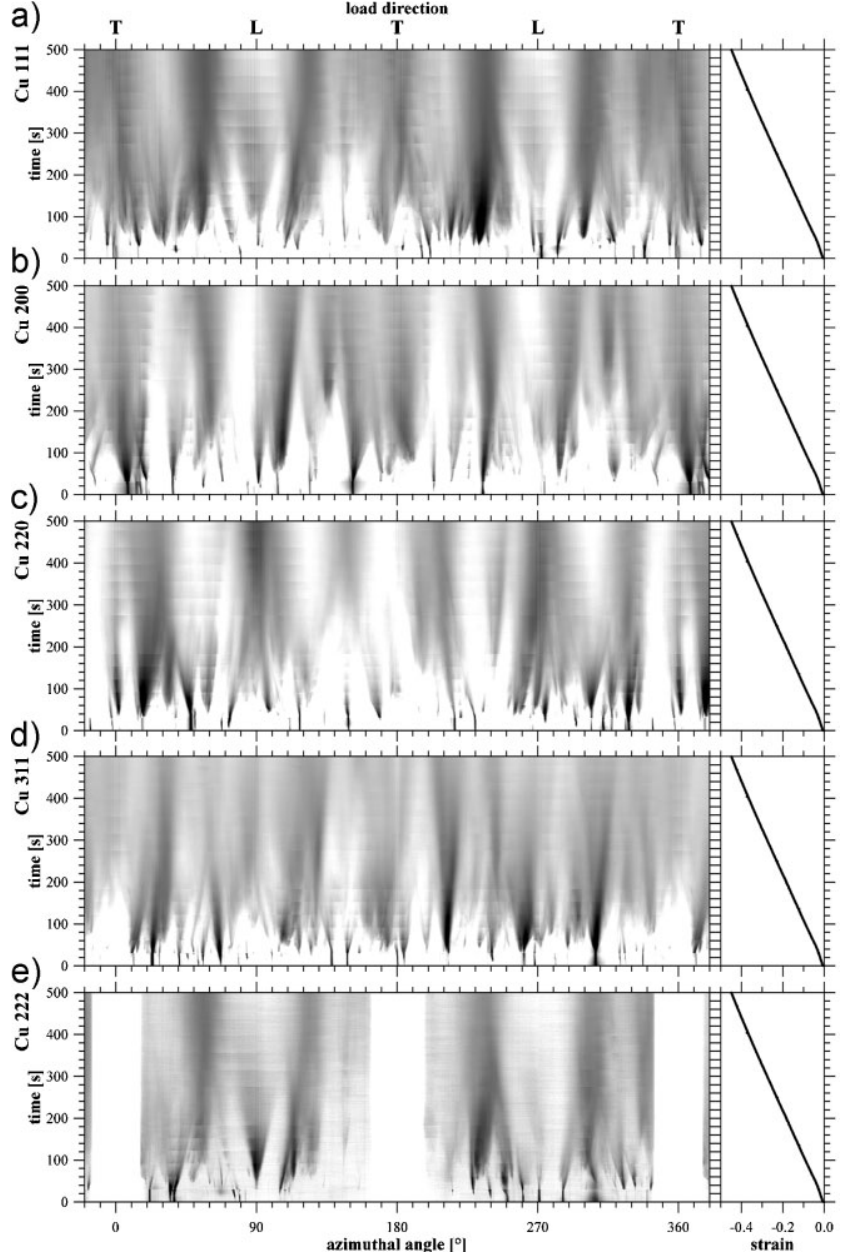

Fig. 3. The intensities of five selected Debye-Scherrer rings are plotted in dependence of azimuthal angle $\gamma$ and with longitudinal $L$ and transverse direction $T$ marked. The range $T \pm 16^{\circ}$ in e) is shaded off due to the limited size of the detector. Engineering strain $\varepsilon$ is shown on the right. Dark is high intensity.

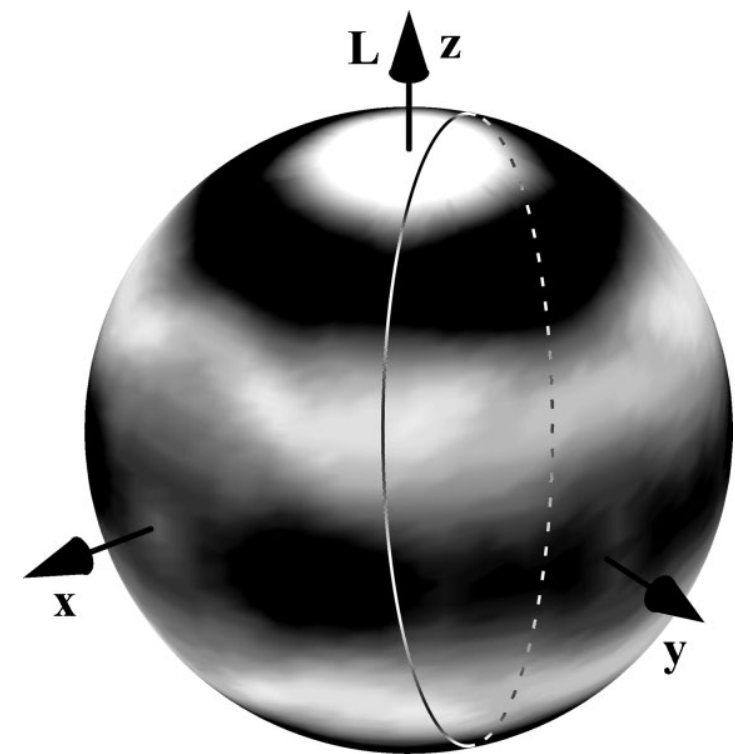

Fig. 4. The reconstructed 111 pole figure from a full texture measurement indicating schematically a line of measurement obtained from one Debye-Scherrer ring, for example, the inner-most ring of Figure $2 b$. Dark is high intensity. 
Figure $2 \mathrm{~b}$ corresponds to the solid-dotted circle on the sphere of Figure 4.

It is interesting to follow the merging of differently oriented grains into the final texture. If grains were arbitrarily oriented, for example, with the (111) plane normal in the $70-80^{\circ}$ and $100-110^{\circ}$ regions in Figure $3 a$, they rotate away from the longitudinal direction into the preferred orientation of $56^{\circ}$. A similar process appears to form the second texture maximum in the $T$ direction at 0 and $180^{\circ}$. Grains which were already oriented close to those directions do not rotate much, however they develop subgrains due to the imposed plastic deformation. In particular, a strong mosaic spread evolves in the $T \pm 56^{\circ}$ directions, indicating slip planes and deformation bands are highly activated. Grains oriented with the normal of (111) planes along the longitudinal stress direction $L$ (90 and $270^{\circ}$ ) are in an unstable equilibrium in which the slip system of that plane is not activated. Therefore, these grains do not rotate. Subgrains, however, are formed through the deformation systems of the other $\{111\}$ planes, creating symmetric mosaic spread of several degrees. Finally, the subgrains take over the role of individual grains and merge into the preferred orientation maxima at $T \pm 56^{\circ}$.

The final texture shown in Figure 4 is not yet axialsymmetric with respect to the uniaxial load axis. However, it is close to the ultimate deformation texture in $\mathrm{Cu}$, which indeed would show axial symmetry. ${ }^{[7]}$ Starting from very anisotropic and arbitrary orientations of a few single grains, the asymptotic texture is approached but not yet achieved, which is expressed in the observed anisotropy. For example, the grains reflecting 111 to the $230^{\circ}$ position or 220 to $18^{\circ}$ are still over-represented after the compression process. In Figure 3, symmetry around the $L$ directions would be necessary for a total axial symmetry. Upon further compression - in the limit of infinite compression, the system should be independent from the starting conditions and evolve to its axial symmetric asymptote.

In summary, it has been demonstrated in an unprecedented way that cold plastic deformation in copper evolves at the level of individual grains. The data obtained from the experimental program provide insight into the detailed evolution of the microstructure during the deformation process. The experiments verified the premise that the deformation behavior of individual grains can be studied in a multi-grained sample. This new experimental technique and the evaluation procedure itself is of generic nature, allowing the study of grain evolutions in almost any crystalline material. Moreover, the technique and analysis can be applied to in situ studies of other related processes that occur during plastic deformation such as dynamic recrystallization. ${ }^{[8]}$ The concept of in situ observations by the use of modern diffraction techniques is still in its infancy in physical thermo-mechanic simulation studies ${ }^{[9]}$ and these techniques should be implemented at synchrotron or neutron facilities in order to provide in-time analysis of deformation processes.

Received: June 4, 2009 Revised: July 22, 2009

[1] K.-D. Liss, A. Bartels, A. Schreyer, H. Clemens, Texture Microstruct. 2003, 35, 219.

[2] P. Landau, R. Z. Shneck, G. Makov, A. Venkert, J. Mater. Sci. 2007, 42, 9775.

[3] L. Margulies, G. Winther, H. F. Poulsen, Science 2001, 291, 2392.

[4] A. Brûlé, O. Kirstein, Physica B 2006, 385, 1040.

[5] U. Garbe, J. Appl. Cryst. 2009, 42, 730.

[6] R. Hielscher, H. Schaeben, J. Appl. Cryst. 2008, 41, 1024.

[7] C. A. Bronkhorst, S. R. Kalidindi, L. Anand, Philos. T. Roy. Soc. A 1992, 341, 443.

[8] K.-D. Liss, U. Garbe, H. Li, T. Schambron, J. D. Almer, K. Yan, Adv. Eng. Mater. 2009, 11, 637.

[9] D. Ferguson, W. Chen, T. Bonesteel, J. Vosburgh, Mater. Sci. Eng. A 2009, 499, 329. 